\section{Summary of: Highly anxious dental patients report more pain during dental injections}

\author{
A. J. van Wijk ${ }^{1}$ and P. C. Makkes²
}

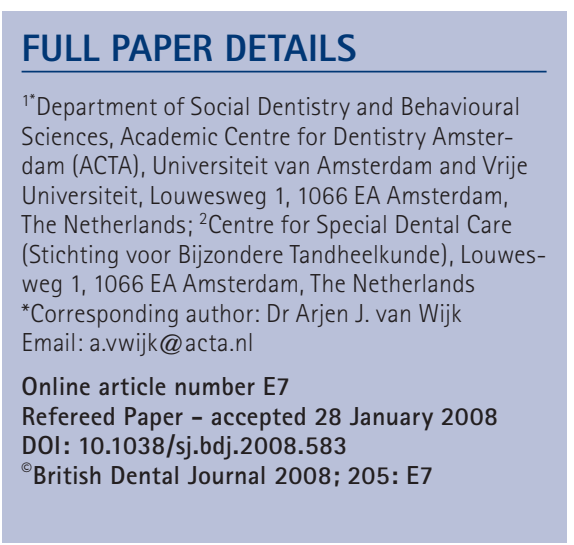

\begin{abstract}
Objectives In the present study, the relationship between anxiety and pain was investigated using a clinically relevant sample and stimulus. Methods A sample of highly anxious dental patients $(n=23)$ and a sample of 'normal' subjects $(n=57)$ were compared with respect to the duration and intensity of pain while receiving a dental anaesthetic injection. Results As expected, highly anxious dental patients indicate more pain, which is of longer duration, than normal patients. Most predictive for the amount of pain felt was the pain felt during a previous injection. Conclusion Dentists should be aware that anxious dental patients with a negative experience regarding dental injections may feel elevated levels of pain which most likely leads to negative expectations for the future.
\end{abstract}

\section{EDITOR'S SUMMARY}

Most dentists will have had experience of a patient who is anxious about dental treatment and some may have treated people who are clearly afraid to visit the dentist. Successful treatment of patients with dental fear can be problematic and any research that can aid our understanding of this condition and provide clues to how best to proceed with treatment in highly anxious patients is obviously of importance.

One aspect of dental treatment that many patients fear is the pain that they believe will be involved and it is the relationship between anxiety and pain that is investigated in this study by van Wijk and Makkes. While there is already a considerable body of research into this relationship, the present study is particularly interesting because it uses a sample (patients treated at a dental fear clinic) and a pain stimulus (dental injections) that are clinically relevant to the dental setting. In this way the results are likely to be representative of the actual experiences of patients with dental fear, as opposed to results obtained using experimental stimuli or that do not use anxious dental patients as a study group.

The authors found that, as expected, highly anxious patients felt more pain, which lasted longer, than normal patients. Interestingly, the biggest predictor for the amount of pain an anxious patient felt was the pain that the patient had felt during a previous injection, showing how previous bad experiences can lead to poor future expectations, increased anxiety and therefore more pain at the next visit. The study clearly highlights the complex and fascinating relationships between pain, fear and dental treatment.

The findings of the paper are useful to dental practitioners in that they may assist them to treat anxious patients. The authors make simple recommendations, such as giving more painful palatinal injections last and using surface anaesthesia to reduce the initial sharp pain of the injection, which are easy to implement. They also show that evaluating treatment with some patients afterwards may help them to reinterpret the experience in a more positive way. By asking the patient about their previous experiences and taking the time and effort to try to reduce their anxiety and pain, the dental visit can be improved for both the patient and the dentist.

The full paper can be accessed from the $B D J$ website (www.bdj.co.uk), under 'Research' in the table of contents for Volume 205 issue 3.

Rowena Milan, Journal Editor

DOI: 10.1038/sj.bdj.2008.681 


\section{TO ACCESS THE BDJ WEBSITE TO READ THE FULL PAPER:}

- BDA members should go to www.bda.org

- Do not login on the BDA home page, if you are already logged in, please log out.

- Then, in www.bda.org click on the link to the BDJ in the top left of the screen. A new window will open. If a new window fails to open please check the settings of any pop up blocker software that you have installed on your computer.

- You will now be asked to login with your BDA website login details which are on your BDA membership card.

- Once your details have been entered you will be transferred to the BDJ website. If your login does not work please contact the BDA Membership Department on 02075634550.

- If you are not able to access the article on the BDJ website there may be an issue with your system's firewall. If so, return to the BDA homepage and click on the link 'BDJ access problems' and follow the step by step guide.

\section{COMMENT}

The findings from van Wijk and Makkes emphasise the importance of examining both pain and anxiety (or fear) in dental and other health settings. The interacting, overlapping and sometimes reciprocal relationships between pain and anxiety (or fear) obviously are of clinical importance, as demonstrated in the present study, consistent with prior data and theoretical formulations.

The highly fearful/phobic patients from the fear clinic provide an excellent sample within which to understand how high degrees of fear influence pain, and vice versa. The comparison sample from a general dental population provides a control group that may be representative of typical dental patients, who likely are on a continuum of dental fearfulness that ranges from fearlessness, to moderate intensity fear, and finally to extreme fear and avoidance.

The dental injection serves as an excellent model for acute pain. Injection fear is one potential aspect of overall dental fear, which also (or instead) can include fears relevant to pain, other specific aspects of the dental situation (eg drilling), catastrophe, disfigurement, or being closedin, as well as being generally fearful or distrustful.

The present study likely focused on fear rather than anxiety. The immediacy of the dental injection and the fact that the patients already were in the dental situation both suggest a fear response, rather than a more cognitive and temporally distal anxiety response. Regardless of the label, however, the importance of the relation with pain is clear.

Future research should focus on the type and degree of invasiveness of the procedure(s) that follow the injection. Present results suggest that expectancy effects drive anticipated fear (which may appropriately be labelled as anxiety); expectancies also determine anticipated pain.

The influence of both prior fear and pain on anticipation of future discomfort in oral healthcare is of critical importance, given the impact on dental attendance/avoidance and timely/ delayed appointments. These findings prompt not simply the question, 'How are anxiety/fear and pain related?' but 'How does the relation between anxiety/fear and pain impact these states in patients of varying characteristics (including degree of anxiety/fearfulness) and in varying situations?'

\section{W. McNeil, Professor, Department} of Psychology/Eberly Professor of Public Service/Clinical Professor, Department of Dental Practice and Rural Health, West Virginia University

B. N. Kyle, Clinical Psychology Doctoral Student, Department of Psychology, West Virginia University

\section{AUTHOR QUESTIONS AND ANSWERS}

1. Why did you undertake this research? A vast amount of literature exists on the relation between anxiety and pain, also within dentistry. However, most of the studies showing increased pain perception as a result of anxiety concern laboratory studies with experimentally induced pain, or artificially created anxious subjects. Therefore, our study made use of a clinically relevant stimulus (ie a dental injection) and truly anxious patients (so anxious that they can only be treated at a dental fear clinic).

\section{What would you like to do next in this} area to follow on from this work?

As we write, a continuation of this study is being carried out among a sample of Turkish patients $(\mathrm{n}=300)$ attending a clinic specialised in implants, extractions and endodontic therapy (complicated cases). Besides measuring the duration and intensity of pain, patients will also fill out the short version of the Fear of Dental Pain questionnaire (FDP) ${ }^{1}$ and a number of other anxiety related measures. Besides establishing the relation between anxiety and pain in this sample of 'normal' patients about to undergo quite invasive treatment, we also hope to find an association between pain felt and FDP score. FDP is an anxiety driven process that leads to overestimated pain and elevated anxiety as a result of this. In the future, we hope to break through the vicious circle of anxiety and overestimated pain by means of interventions tailored to the patients needs.

1. van Wijk A J, McNeil D W, Ho C J, Buchanan H, Hoogstraten J. A short English version of the Fear of Dental Pain questionnaire. Eur J Oral Sci 2006; 114: 204-208. 